\title{
Patrón Alimentario y Estado Nutricional en Niños Desplazados en Piedecuesta, Colombia
}

\section{Displaced and local children's alimentary patterns and nutritional state in Piedecuesta, Colombia}

\author{
Edna M. Gamboa-Delgado, Nahyr López-Barbosa, \\ Lina M. Vera-Cala y Gloria E. Prada-Gómez
}

Departamento de Salud Pública, Escuela de Medicina y Escuela de Nutrición y Dietética, Universidad Industrial de Santander. Bucaramanga, Colombia. magalygamboa@yahoo.com, nlopez@uis.edu.co, linamavc@yahoo.com,gprada@telebucaramanga.net.co

Recibido 1 Agosto 2006/Enviado para Modificación 20 Enero 2007/Aceptado 10 Febrero 2007

\section{RESUMEN}

Objetivos Determinar el patrón alimentario en la población de niños en edad escolar del sector Guatiguará (Piedecuesta), evaluar su estado nutricional y la asociación con los factores socioeconómicos y alimentarios.

Metodología Estudio descriptivo de corte transversal en 89 niños desplazados y no desplazados en edad escolar (6 a 12 años) del sector Guatiguará (población: 258), realizado en 2004. Se aplicó una Encuesta Sociodemográfica y Nutricional y un Formato de Consumo de Alimentos (SICl); Se utilizó el método recordatorio de 24 horas, juegos de menaje y gráficas representativas del tamaño de porción de alimentos. Se tomó el peso y la talla de los niños. Procesamiento en programas $\mathrm{SICl}$, Excel, Epi Info 6.04d y Stata 8.0.

Resultados Estado Nutricional (P/E): Déficit 25,8 \% y Riesgo de Déficit 14,6\%; déficit T/E 25,7\% y déficit P/T 4,3\%. Se observa un bajo consumo de verduras, frutas y alimentos proteicos. Los porcentajes más bajos de adecuación del consumo correspondieron a calcio, vitamina B6, hierro, cinc y niacina. Se encontró Asociación entre recibir ingresos familiares mensuales menores de $\$ 150000$ y déficit nutricional (OR=2,76 IC(95\%) 0,92-8,44, $p=0,040)$. Factores protectores para déficit nutricional: ser madre empleada (OR=0,29, IC(95\%) 0,09-0,84, p=0,012) y familias con 2 o 3 miembros trabajadores y aportantes a los gastos del hogar (OR=0,17 IC (95\%) 0,03-0,69 $p=0,005$ ).

Conclusión En la población de estudio, el factor socioeconómico es determinante del patrón alimentario y del estado nutricional.

Palabras Clave: Estado nutricional, patrones alimentarios, población vulnerable (fuente: DeCS, BIREME). 


\section{ABSTRACT}

Objectives This study was aimed at establishing displaced and local children's alimentary patterns amongst the school-age population from the Guatiguará area (Piedecuesta) and correlating their nutritional state with chosen socioeconomic and nutritional factors.

Methods This descriptive transversal study of 89 displaced and local school-aged children (6 to 12 years of age) from the Guatiguará area (population=258) was carried out in 2004. Data was collected from socio-demographic and nutritional surveys as well as a food intake questionnaire ( $\mathrm{SICl}$ ). Twenty-four hour dietary recall, standardised kitchen item games and photographic models of the size of food portions were also used. The children's height and weight were measured. SICl, Excel, Epi Info 6.04d and Stata 8.0 software were used for processing the data.

Results Nutritional state (weight per age - W/A) was as follows: $25,8 \%$ deficit and $14,6 \%$ risk of deficit. Low vegetable, fruit and protein consumption was found. The lowest percentages regarding consumption of needed items pertained to calcium, vitamin B6, iron, zinc and niacin. The relationship between receiving a monthly family income of less than $\mathrm{Col} \$ 150000$ and nutritional deficit was $(\mathrm{OR}=2.76 \mathrm{Cl}(95 \%)$ $0,92-8,44, p=0,040)$. Nutritional deficit protection factors consisted of having an employed mother (OR=0.29 Cl(95\%) 0,09-0,84, $p=0,012)$ and families having 2 or 3 employed members covering household expenses (OR=0.17 Cl(95\%) 0,03-0,69 $\mathrm{p}=0,0052)$.

Conclusion Selected socioeconomic factors influenced the studied population's alimentary pattern and nutritional state.

Key Words: Nutritional status, feeding pattern, vulnerable population (source: $\mathrm{MeSH}$, $N L M)$.

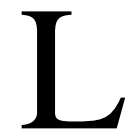

a nutrición es un derecho fundamental y aunque la Convención de los Derechos del Niño afirma que "Todos los niños y las niñas tienen el mismo derecho a una alimentación suficiente y adecuada", la realidad muestra que en condiciones de pobreza y desplazamiento este derecho no se cumple generando un deterioro del bienestar infantil (1).

Según datos presentados por la Presidencia de la República, en el año 2000, la población en nivel de pobreza por ingresos fue de 59,8 \% disminuyendo a 58,8 \% en el 2001, mientras que el nivel de indigencia pasó de $18,1 \%$ a 23,4 \% en 1997 y 2000 respectivamente $(1,2)$, entre 1985 y 1999 en Colombia fueron desplazados por la fuerza alrededor de 392000 hogares que representan aproximadamente 1900000 personas, de las cuales 1100000 son menores de edad. Desde enero de 2000 a junio de 2001, Santander ocupó el puesto 11 como lugar de expulsión con un 24,4 \% del total de la población desplazada a nivel nacional y un 3,1\% como lugar de llegada. 
El desplazamiento forzado afecta la salud y el bienestar de las familias, y adquiere mayor gravedad cuando involucra niños, niñas y jóvenes que desde una condición de alta vulnerabilidad, viven situaciones de violencia que lesionan sus derechos $(3,4)$, por otra parte los lugares de asentamiento, por lo general, son los más pobres y no están preparados para recibir a los nuevos habitantes, empeorándose con ello la carencia de servicios básicos para las comunidades receptoras. Lo anterior contribuye al deterioro del estado nutricional, situación que se refleja en las altas prevalencias de desnutrición $(5,6)$.

Según el diagnóstico de la situación de salud de las poblaciones desplazada y receptora, la Desnutrición Crónica en el municipio de Piedecuesta fue de 42,9 \%, mientras la Desnutrición aguda se presentó en un 28,6 \% y la Desnutrición global en un 50,0 \% (7).

Al no existir estudios relacionados con los patrones de consumo y con el estado nutricional de la población en edad escolar de este sector, se hace necesario realizar un diagnóstico que permita determinar el patrón alimentario e identificar el estado nutricional, para que sirva de base en la planeación y ejecución de intervenciones alimentarias y nutricionales a favor del bienestar y la salud de éste grupo vulnerable. El objetivo de este estudio es determinar el patrón alimentario y evaluar el estado nutricional y la asociación con los factores socioeconómicos y alimentarios en la población de niños en edad escolar del sector Guatiguará del Municipio de Piedecuesta.

\section{MATERIALES Y MÉTODOS}

En el segundo semestre de 2004, se llevó a cabo un estudio de corte transversal descriptivo, en una población de 258 niños en edad de 6 a 12 años, del sector Guatiguará del municipio de Piedecuesta. En este estudio se evaluaron niños desplazados por la violencia y niños que no lo son, pero que sin embargo, viven en ese sector. Se calculó un tamaño de muestra de 89 niños, basado en una prevalencia del $50 \%$, precisión del 8,5\%, poder del $80 \%$ y nivel de confianza del $95 \%$.

A través de entrevista directa se aplicó una encuesta sociodemográfica a las madres de los 89 niños participantes en el estudio, mientras que el consumo de alimentos sólo fue posible evaluarlo en 76 de ellos.

El consumo de alimentos de los niños, fue obtenido mediante el método recordatorio de 24 horas (R24H) aplicado a las madres, por ser éstas quienes 
poseen mayor información acerca del consumo alimentario de sus hijos y para garantizar una mayor veracidad en cuanto al reporte de las cantidades de alimentos. La información sobre la alimentación consumida por los niños se basó en el reporte de un día de alimentación normal, en casa. Para obtener información del tamaño de porción de las preparaciones consumidas por los menores, se utilizaron juegos de menaje y gráficas representativas del tamaño de la porción de preparaciones de alimentos que corresponden a instrumentos estandarizados del programa SICI 2,0 (8). Los encuestadores tomaron el peso y la talla a los niños participantes.

Se recolectó información en población cautiva (en las aulas escolares más cercanas al sector) y también directamente en la residencia de los niños.

Todos los registros (R24H) fueron digitados por duplicado y validados en EpiInfo v 6,04 d (9). El consumo total de cada nutriente se calculó como la sumatoria del producto de la cantidad de alimento consumido por el contenido del nutriente; se utilizó el programa SICI v 2,0 para realizar este cálculo (8). La ingesta actual de macro y micronutrientes fue comparada con los valores de referencia para Colombia (recomendaciones poblacionales del Instituto Colombiano de Bienestar Familiar, ICBF) (10,11). La adecuación de la ingesta (razón entre ingesta estimada y la recomendada) fue expresada como un porcentaje. Además, se estableció la participación relativa de los macronutrientes al consumo total de calorías diarias estimadas por el R24H (valor calórico Total VCT).

Se utilizaron los programas Epi Info v 6,04d y Stata v 8,2 (12) para generar estadísticas univariadas, en las que se presentan las variables continuas con sus medidas de tendencia central y sus correspondientes medidas de dispersión y las variables categóricas con sus correspondientes frecuencias. Se realizó un análisis bivariado entre los factores determinantes más relevantes y el estado nutricional de los niños.

El presente trabajo tuvo en cuenta los principios fundamentales de la ética: autonomía, beneficencia, no maleficencia y justicia (13). Según la Resolución 8430 de 1993, esta investigación no representa ningún riesgo para los niños en estudio. Las madres de los menores firmaron el consentimiento informado, mediante el cual conocieron los procedimientos a los que se someterían ellas y sus hijos y los beneficios que representa su participación en el estudio (14). 


\section{RESULTADOS}

\section{Condiciones Sociodemográficas y de salud}

Guatiguará es una vereda del municipio de Piedecuesta en constante crecimiento, habitada por familias de muy bajos ingresos; gran parte de esta comunidad es víctima del desplazamiento forzado. En la Tabla 1, se presentan las principales características sociodemográficas de la población estudiada. Una tercera parte de los padres y dos terceras partes de las madres están desempleados. La jefatura del hogar estuvo a cargo del padre en el 59,6 \% de los casos y de la madre en el 15,7 \%. En cuanto al nivel de escolaridad una tercera parte de los padres y madres completó la primaria y sólo el 4,5 \% de los padres completó el bachillerato.

Tabla 1. Características sociodemográficas de la muestra Piedecuesta. Santander. 2004

\begin{tabular}{|c|c|}
\hline Características & Total $n=76^{a}$ \\
\hline Edad (años) & $9.02(8,6 ; 9,5)^{b}$ \\
\hline Sexo Femenino & $41\{46,1\}^{c}$ \\
\hline Ingresos superiores a $\$ 150000$ & $43\{48,3\}$ \\
\hline Familias con 5 o más miembros & $64\{71,9\}$ \\
\hline $\begin{array}{l}\text { Solo un integrante de la familia trabaja y } \\
\text { aporta a los gastos del hogar }\end{array}$ & $68\{76,4\}$ \\
\hline
\end{tabular}

Patrón alimentario. Un 50 \% de los niños no ingieren refrigerio en la mañana y 84,2 \% no lo consumen en la tarde. El 4,5 \% recibe algún tipo de complemento nutricional en sus familias.

La compra de alimentos, perecederos y no perecederos, se realiza con mayor frecuencia en las tiendas de la vereda; predomina la frecuencia de compra de alimentos diaria. El valor promedio del gasto familiar mensual en alimentos es de $\$ 151011$ (DS= \$ 87 992), equivalente a un 0,39 Salario Mínimo Mensual Legal Vigente (SMMLV), con valores desde \$5 000 hasta $\$ 450000$. En la mitad de las familias, la madre es la responsable de la compra de alimentos.

Dentro de los alimentos básicos que hacen parte del consumo infantil, los de mayor frecuencia de consumo fueron la panela en un $90,7 \%$, la papa en un $86,4 \%$, el arroz blanco en un 75,0 \%, el aceite en un 73,6\%, el tomate en un $64,4 \%$ y la harina de cereales, al igual que el pan, en un 57,8\%. Los alimentos consumidos en mayor proporción son de tipo energético, mientras los alimentos 
fuente de proteína de origen animal sólo son consumidos por la mitad de los niños (leche 51,3 \%, huevo 47,3 \% y carne 40,7 \%). Menos del $20 \%$ de los niños consume proteína vegetal, siendo su fuente principal las leguminosas secas (18,4 \% consume fríjol, 15,7 \% lenteja y 18,4 \% arveja seca). De las frutas, la de mayor consumo es el limón (53,9 \%) y de las verduras el tomate (64,4 \%), seguido por la zanahoria (38,1\%).

Además se presentó un consumo considerable de frescos (saborizantes) y gaseosa $(31,5 \%)$.

En cuanto a las preparaciones consumidas en cada tiempo de comida, para el desayuno las más comunes fueron: agua de panela (61,8\%), caldo de papa (36,8 \%), pan (34,2 \%) y arepa (30,2 \%); en el almuerzo: arroz seco (67,1 \%), papa, yuca o plátano cocido (53,9 \%), limonada (44,7 \%) y carne $(27,6 \%)$ y durante la comida: agua de panela (44,7 \%), pan (31,5\%), arroz seco (18,4\%) y papa, yuca o plátano (14,4\%). En el desayuno existe una deficiencia del 100 \% en el consumo de frutas y sólo $25 \%$ de los niños consume alimentos fuentes de proteína (huevo). En el almuerzo se observa deficiencia en el consumo de verduras en el $90 \%$ de los casos, de frutas en el $75 \%$ y de alimentos proteicos en el $50 \%$, mientras el consumo de saborizantes alcanza casi el $20 \%$. La última comida del día, presenta las mayores deficiencias, siendo nulo el consumo de verduras en el $100 \%$ de los casos y de alimentos proteicos y frutas en el 90 $\%$.

Adecuación entre lo consumido y lo requerido. El nivel de adecuación media entre lo consumido y lo recomendado para estos niños, según el ICBF (11), mostró que, para 10 de 13 nutrientes evaluados, el porcentaje de adecuación fue inferior a $100 \%$, lo mismo que para la adecuación energética (65,0 \% de adecuación media). De las vitaminas y minerales, los de menor porcentaje de adecuación fueron la vitamina B6 y el calcio (51,6 \% y 42,5 \% respectivamente).

Estado nutricional. El 25,8 \% presentó déficit, según el Peso para su edad; el 78,3 \% de éstos son hombres $(\mathrm{p}=0,004)$. Se encontró un 4,3\% de niños con déficit de Peso para la Talla $(\mathrm{P} / \mathrm{T})$ y un $25,7 \%$ de menores con déficit de Talla para la Edad (T/E).

De los niños pertenecientes a hogares que reciben ingresos mensuales menores de \$150 000 (0,38 SMMLV), el 59,1 \% presenta algún tipo de déficit nutricional, mientras que entre los que forman parte de hogares con ingresos mensuales iguales o superiores a \$150 000, esta proporción se encontró en $34,3 \%(p=0,040)$. 
Del total de niños a riesgo de desnutrición, el 61,5 \% son hijos de mujeres sin empleo y del total de niños desnutridos este porcentaje aumentó al 91,3 \% $(p=0,092)$. Entre los niños pertenecientes a hogares en los cuales sólo un miembro de la familia trabaja y aporta a los gastos de la casa, el 48,5 \% presenta algún grado de déficit nutricional, mientras que donde 2 o más miembros de la familia trabajan y aportan, el porcentaje corresponde a $14,3 \%(p=0,005)$.

El $50 \%$ de los niños, cuyas madres son amas de casa, presentan algún grado de déficit nutricional, mientras que ese mismo tipo de deficiencia se presenta en un 27,0 \% de los niños cuyas madres tienen una ocupación diferente $(\mathrm{p}=0,030)$.

El 41,9 \% de los hijos de mujeres con bajo nivel de escolaridad, presentó algún grado de déficit nutricional, en contraste con un 33,3 \% de ese mismo déficit presentado en los hijos de mujeres con mayor escolaridad $(p=0,538)$.

El sexo masculino resultó asociado al estado de déficit nutricional $(\mathrm{OR}=4,57$ IC (95 \%) 1,65-13,16 p=0,001), así mismo se encontró asociación entre el hecho de recibir ingresos familiares mensuales menores de $\$ 150000$ y el evento déficit nutricional (OR=2,76 IC(95 \%) 0,92-8,44, p=0,040). Las madres empleadas constituyen un factor protector para el déficit nutricional de los menores (OR=0,29 IC (95\%) 0,09-0,84 $\mathrm{p}=0,012$ ). Pertenecer a familias que cuentan con 2 o 3 miembros que trabajan y aportan a los gastos del hogar, también mostró ser un factor protector para el déficit nutricional de los menores $(\mathrm{OR}=0,17$ IC $(95 \%)$ 0,03-0,69 p=0,0052). Aunque se encontró asociación entre tener más de 5 años de escolaridad aprobados por la madre y el déficit nutricional de sus hijos (OR=0,69 IC (95\%) 0,16-2,51, p=0,530), ésta no resultó ser estadísticamente significativa.

\section{DISCUSIÓN}

Acceso a los alimentos. En Colombia, como en otros países de América Latina, la accesibilidad a los alimentos depende de los ingresos para adquirir una canasta de alimentos que permita satisfacer sus necesidades nutricionales. El patrón alimentario y el estado nutricional se ven afectados por el desplazamiento forzado y la pobreza, debido a que el reducido ingreso familiar limita la compra y consumo de alimentos (15).

Una Canasta Básica Alimentaria (CBA) es definida como el conjunto de alimentos representativos del consumo poblacional en cantidad suficiente para 
cubrir la necesidad de energía de una familia promedio (16). En los países en desarrollo la seguridad alimentaria se ve afectada por las limitaciones económicas debido al reducido ingreso familiar que limita la compra y el consumo de alimentos (15). Las familias estudiadas gastan en alimentos en promedio 0,37 del SMMLV para el año de 2006 y una CBA normativa costaba 1,1 SMMLV en el 2005 en el mismo municipio, por tanto, los recursos con que disponen estas familias no permiten acceder a una nutrición adecuada (17).

La compra de alimentos se realiza principalmente en la tienda, al igual que en otros estudios $(18,19)$, las razones que justifican este comportamiento son el fraccionamiento de los productos alimentarios, adaptado a la capacidad de compra y la cercanía de la tienda al hogar $(20,21)$.

Patrón alimentario. El consumo de alimentos como arroz y panela es mayor al encontrado en otros grupos vulnerables no desplazados (22), debido a su bajo costo y a las limitaciones del presupuesto familiar. La fuente proteica animal se cubre a expensas del huevo, alimento percibido por la comunidad como la "proteína de los pobres" (23), situación similar a la encontrada en otros estudios con población desplazada (19). La cantidad consumida de leche es mínima, pues aunque este alimento hace parte del consumo del 51,3 \% de los niños, sólo se utiliza como ingrediente de otras preparaciones.

Adecuación de la dieta. Los promedios de los porcentajes de adecuación de consumo más bajos se presentaron en los micronutrientes Calcio, vitamina B6, hierro, cinc y niacina. En Colombia según el Instituto Nacional de Salud (INS) existe deficiencia de vitamina A, y esta se calificó como un problema de salud pública (24). La deficiencia de cinc es común en los países en vía de desarrollo (25), afecta el crecimiento de los menores y en general la composición corporal (26), y juega un papel importante en la respuesta inmune en adultos y niños (27). De manera similar, la deficiencia de hierro encontrada en este estudio concuerda con la tendencia al aumento que ha presentado la anemia en menores de cinco años, durante los últimos años (prevalencia de anemia: $23,4 \%$ en 1995 y 32,2 \% en 2005) (24).

Estado nutricional. La prevalencia de desnutrición es indicador de impacto negativo sobre la población infantil y refleja la presencia de factores que inciden negativamente en el desarrollo de niños y niñas (bajo peso al nacer, falta de lactancia materna, pautas erróneas de alimentación infantil, enfermedades frecuentes, condiciones higiénicas desfavorables, ingesta nutricional deficiente 
y bajo nivel educativo de las madres), todas ellas condiciones asociadas con la pobreza y el bajo nivel educativo de los padres.

En este estudio se observa que frente a algún grado de déficit para el indicador nutricional Peso/Edad, son factores de riesgo: ser sexo masculino y tener ingresos mensuales familiares menores de 0,38 SMMLV (\$150 000) y son factores protectores: tener madre empleada y pertenecer a familia con $2 \mathrm{o}$ 3 miembros que trabajan y aportan a los gastos del hogar. En la población de estudio, el factor socioeconómico es determinante del patrón alimentario y del estado nutricional. La escolaridad parece ser factor protector, como lo mencionan Smith y Haddad (28), pero probablemente por falta de sujetos en la muestra, la asociación no resultó estadísticamente significativa en nuestro estudio.

Como conclusiones se evidenció un bajo consumo de verduras y frutas y un alto consumo de saborizantes (fresco, gaseosa), comportamiento que puede modificarse hacia el consumo de jugos de frutas naturales. Como práctica alimentaria positiva se observa el consumo de leguminosas secas, las cuales deben ser un recurso para cubrir las necesidades de este nutriente en poblaciones con bajos ingresos económicos (29).

Los hallazgos de este estudio sugieren que las madres de los niños analizados requieren capacitación y asesoría nutricional respecto a la compra y preparación de alimentos saludables, especialmente frutas y verduras. De igual manera es preciso promocionar, en esta comunidad, el desarrollo de proyectos que impulsen las competencias laborales y la productividad de sus habitantes, con el fin de elevar los ingresos familiares y de esta manera contribuir positivamente a la calidad alimentaria de la familia.

La información obtenida mediante este estudio hace una contribución científica a la Salud Pública, dado que los datos presentados facilitarán el diseño, ejecución y evaluación de estrategias de intervención basadas en evidencia científica, dirigidas a mejorar las condiciones de salud y la calidad de vida del grupo poblacional estudiado*

Agradecimientos. A los estudiantes Liliana Vega, Claudia Johanna Angel, Francia Carolina Espitia, Jenny Landínez, Hamara Páez, Dora Liliana Prada, Wilhem Alfaro, Jordana Ramírez y Viviana Suárez. A la Escuela Popular Claretiana y al Colegio de Promoción Social de Piedecuesta. 


\section{REFERENCIAS}

1. UNICEF. Por una niñez bien nutrida: Comunicación para la Acción, Convención de los Derechos de los Niños, CDN. Art. 24 UNICEF Colombia; 2004. p. 126.

2. Centro de noticias del estado. Presidencia de la República. Colombia, 2003. [Internet]. Disponible en: http://www.presidencia.gov.co/cne/2003/agosto/13/22132 003.htm Consultado Enero 24 de 2007.

3. El desplazamiento forzado: fuentes, causas y distribución geográfica. Observatorio de los derechos humanos en Colombia. 2000. [Internet]. Disponible en: http:/ /www.derechoshumanos.gov.co/observatorio/04_publicaciones/04_01_bole tine s/04_boletin_12/cifras12.htm Consultado Enero 24 de 2007.

4. UNICEF. Ciudades para la Niñez: Los derechos de la infancia, la pobreza y la administración urbana. UNICEF Colombia; 2004. p.340.

5. Rojas-Rodríguez J. Esta Guerra no es Nuestra y la estamos perdiendo. UNICEF Colombia yCODHES; 2000. p. 72.

6. Herrán OF, Herrera M, Amorocho M, Prada GE. Atlas del Estado de Nutrición en Santander. Bucaramanga, Colombia: UIS/ICBF/OPS; 2000.

7. Organización Panamericana dela Salud. Serie Salud y Desplazamiento en Colombia. Comparación de la situación de salud, entre población en situación de desplazamiento y receptora, en seis ciudades. 2002-2003, Modulo 3. Bucaramanga: OPS; 2005.

8. Prada GE. Sistema de Información para Cuantificar la Ingesta. SICI versión 2.0. Bucaramanga, Colombia; 2001.

9. CDC. EpiInfo, versión 6.04d. Epidemiología en ordenadores. Atlanta, Georgia;. Enero2001.

10. Centro de atención nutricional. Tabla de composición de alimentos. Segunda edición. Medellín; 2001.

11. Instituto Colombiano de Bienestar Familiar. ICBF. Recomendaciones de consumo diario de calorías y nutrientes para la población Colombiana. Bogotá: ICBF; 1992.

12. Stata Corp. Stata Statistical Software: Release 8.2. College Station, TX: Stata Corporation; 2003.

13. Galindo SB. Aspectos éticos en la investigación con seres humanos. MEDICAS UIS. 1999; 18 (1): 65-66.

14. Ministerio de Salud República de Colombia. Resolución 008430 de 1993. Bogotá; 4 octubre de 1993.

15. Torres F, Trápaga Y, Delgadillo J, Oceguera D, Zamora J, García JC, et al. La alimentación de los mexicanos en la alborada del tercer milenio México: IIEcUNAM; 2004. 
16. Herrán OF, Prada GE. Determinación de la canasta básica alimentaria de dos municipios colombianos. Salud UIS. 1999; 30: 22-29.

17. Herrán OF, Prada GE, Patiño GA. Canasta básica alimentaria eíndice de precios en Santander, Colombia, 1999-2000. Salud Pública México. 2003; 45:35-42.

18. Programa Mundial de Alimentos. Estudio de caso de las necesidades alimentarias de la población desplazada de Colombia. Junio. 2001. [Internet]. Disponible en: http://www.disasterinfo.net/desplazados/informes /pma/ FinalReportSpanish.htm Consultado Enero 24 de 2007.

19. Prada GE, Herrán OF. Calidad de la dieta en mujeres con condición de desplazamiento forzado. Colombia, 2004. Revista Chilena de Nutrición. 2006; 33(1):22-30.

20. Schejtman A. Economía política de los sistemas alimentarios en América Latina. Santiago de Chile: FAO, 1994; 1993.

21. Morón C, Schjtman A. Evolución del consumo de alimentos en América Latina. En: Producción y manejo de datos de composición química de alimentos en nutrición. Chile: Universidad de Chile. FAO; 1997.

22. Herrán OF, Prada GE. Situación alimentaria en mujeres de 2 municipios Santandereanos. Salud UIS. 2001; 33:134-141.

23. Prada GE. Percepción social de la alimentación en la zona de intervención del programa CARMEN, Bucaramanga. Universidad Industrial de Santander. Reporte Técnico. Bucaramanga. Colombia; 2003.

24. Castro de Navarro LC, Nicholls S. Deficiencia de hierro, vitamina A y prevalencia de parasitismo intestinal en la población infantil y anemia nutricional en mujeres en edad fértil, Colombia 1995-1996. Ministerio de Salud. Instituto Nacional de Salud. Santafé de Bogotá; 1998.

25. Rosado JL. Zinc and copper: proposed fortification levels and recommended zinc compounds. J Nutr 2003;133:2985S-2989S.

26. Rosado JL, López P, Muñoz E, Martínez H, Allen LH. Zinc and iron supplementation do not affect growth, but improve body composition and reduce morbidity of growth-stunted Mexican preschoolers. Am J Clin Nutr 1997;65:13-19.

27. Bhandari N, Bahl R, Hambidge KM, Bhan MK. Increased diarrhoeal and respiratory morbidity in association with zinc deficiency-a preliminary report. Acta Pediatr 1996;85:148-150.

28. Smith L, Haddad L. Research Report 111, Instituto Internacional de Investigaciones sobre Políticas Alimentarias (IFPRI), Washington, D.C.; 2000.

29. Prada GE, Soto A, Herrán OF. Consumo de leguminosas secas en el Departamento de Santander. Colombia. 2002-2003. ALAN 2005; 55(1):64-70. 